\title{
Birefringence and polarization rotation in resonant $x$-ray diffraction
}

\author{
Yves Joly, ${ }^{1,}{ }^{*}$ S. P. Collins, ${ }^{2}$ Stéphane Grenier, ${ }^{1}$ Helio C. N. Tolentino, ${ }^{1}$ and Maurizio De Santis ${ }^{1}$ \\ ${ }^{1}$ Institut Néel, CNRS \& Université Joseph Fourier, BP166, F-38042 Grenoble Cedex 9, France \\ ${ }^{2}$ Diamond Light Source Limited, Diamond House, Harwell Science and Innovation Campus,
}

Didcot, Oxfordshire, OX11 ODE, United Kingdom

(Received 27 June 2012; published 7 December 2012)

\begin{abstract}
Birefringence can contribute to x-ray resonant Bragg diffraction and likely explains recent novel data collected on $\mathrm{CuO}$. We prove these statements using ab initio simulations which reproduce the experimental polarization effects quantitatively. We show that an unrotated polarization signal-ruled out in resonant magnetic scattering within the electric dipole approximation — arises from the dynamic change in polarization inside the material. We are able to reproduce all the related behavior with circular polarization and its dependence on the angle of rotation about the Bragg wave vector. We provide a tool to disentangle the various physical origins of the polarization rotation, providing a more complete understanding of the illuminated material.
\end{abstract}

PACS number(s): 78.20.Bh, 61.05.cj

Polarization phenomena in photon scattering, from optics to $\mathrm{x}$ rays, ${ }^{1}$ provide information on electronic degrees of freedom not available with any other technique in material science. In the past decade, striking progress has been made with polarization analysis-using polarization in both the primary and deflected photons - in experiments performed at synchrotron sources that produce intense, highly collimated and tunable beams of soft or hard $\mathrm{x}$ rays, none more so than in resonant Bragg diffraction with the intensity of a Bragg spot enhanced and labeled ion specific, all achieved by matching the photon energy to an atomic resonance of a selected ion while conditions demanded for Bragg diffraction are enforced. By this technique weak Bragg spots yield unique and valuable information about electron spin and orbital properties that partake in magnetism, superconductivity, and multiferroic behavior, for example.

Birefringence, in which light is divided by double refraction into two components, has not been accounted for in previous interpretations of resonant diffraction from atomic motifs since, unlike the case of soft $x$-ray reflectivity, ${ }^{2}$ the data are conventionally interpreted within the framework of kinematical diffraction. It is then assumed that the polarization is modified only by the scattering process and that the material through which the beams pass, before and after scattering, serves only to give an overall attenuation. Since birefringence at energies close to absorption edges (the conditions under which resonant diffraction takes place) has been shown to be very significant, ${ }^{3-6}$ this assumption must be challenged. This is especially true when small changes to the expected polarization dependence may motivate an exotic explanation of the scattering process. For example, within the electric dipole approximation, the emerging x-ray linear polarization is entirely rotated, with any nonrotated component indicating some higher-order scattering process. Similarly, any dependence on circular polarization can be seen as a fingerprint either of an exotic atomic scattering event or a noncollinear magnetic structure. Birefringence and linear dichroism, which are related via a Kramers-Kronig transform, modify both the polarization and phase of the waves and thus render both of these assumptions void (see Fig. 1).

Since both exotic resonant scattering and birefringence (within the electric dipole approximation) are permitted by symmetry in $\mathrm{CuO}$, only ab initio calculations can give a reliable quantitative description of the scattering signals, and determine the dominant physics. This is the purpose of the present Rapid Communication.

$\mathrm{CuO}$ crystallizes in the monoclinic space group $C 12 / c 1$ (No. 15) ${ }^{7}$ and, in common with all noncubic crystals, exhibits birefringence at absorption edge resonances. The material is multiferroic just below the paramagnetic transition at $T_{N 2}=$ $230 \mathrm{~K}$ and above $T_{N 1}=213 \mathrm{~K}^{8}$ Below $T_{N 1}$ it is an antiferromagnet with an ordering wave vector $\mathbf{q}=(1 / 2,0,-1 / 2) .{ }^{9,10}$ In a recently reported experiment, ${ }^{11}$ the magnetic $(1 / 2,0,-1 / 2)$ reflection was recorded at $100 \mathrm{~K}$, under various conditions of the incoming polarization, with an analysis of the outgoing polarization and with photon energy close to the $\mathrm{Cu} L_{23}$ edge. Observations of a nonrotated polarization component and a strong dependence on the circular polarization of the incident beam guided the authors to the exciting possibility of electric dipole-magnetic dipole (E1M1) scattering from an orbital or toroidal current around the scattering copper atoms, supporting the recent suggestion that such multipoles play a role in high-temperature superconductivity in cuprates. ${ }^{12}$ Moreover, Wu et al. ${ }^{13}$ measured the same reflection in $\mathrm{CuO}$ at a single energy just below the $L_{3}$ edge and attributed the observed nonrotated polarization intensity to a memory effect in the multiferroic transition through $T_{N 1}$ as a result of preserving the handedness of spin spirals in multiferroic nanoregions.

An alternative explanation for these results is found in birefringence and dichroism. Indeed, the electric dipole atomic magnetic scattering gives only a polarization-rotated contribution ( $\sigma \pi^{\prime}$; see Fig. 1). However, as the beam propagates through the crystal, the incoming $\sigma$ wave is rotated slightly and becomes elliptical, leading to an apparent $\sigma \sigma^{\prime}$ contribution. Only a quantitative evaluation can disentangle the relative importance between these effects and more exotic E1M1 or $E 1 E 2$ (dipole-quadrupole) resonant scattering. For this purpose we used the approach developed by Collins and Lovesey ${ }^{14}$ and Palmer, ${ }^{3}$ and included it in the FDMNES code. ${ }^{15}$

First, even without considering birefringence, absorption itself has a significant effect and must be taken into account. This is done by considering the scattering from a thin layer, located at a depth $z$ beneath the flat crystal face. The length of the material along which the incident $(i)$ and scattered 


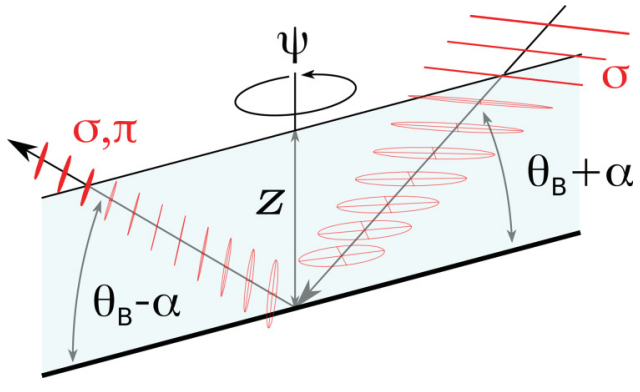

FIG. 1. (Color online) The propagation of polarized waves within the sample. Here, the incident polarization is perpendicular to the scattering plane $(\sigma)$ as it enters the sample. By the time the beam scatters from a layer of the sample at depth $z$, its polarization is a rotated ellipse by the effects of birefringence and dichroism. The polarization state continues to evolve until the beam emerges from the sample.

(s) beams propagate is then $l_{i(s)}=z / \sin \left[\theta_{B}+(-) \alpha\right]$. Integration over $z$ is simple and yields the result that the uncorrected intensity must be divided by the factor $a \bar{\mu}$ with $a=1 /\left[\sin \left(\theta_{B}-\alpha\right)+\sin \left(\theta_{B}+\alpha\right)\right]$. Two further corrections may be applied. The first is a factor $e^{-a \bar{\mu} \delta}$ that accounts for a nondiffracting (e.g., disordered) surface layer of the same material, of thickness $\delta$. The second is a further factor $1 / \bar{\mu}$ that gives an approximate correction in the case that the peak height is measured rather than the peak area. This takes into account the diffracting thickness, inversely proportional to $\bar{\mu}$, which is, also in the present case, smaller than the longitudinal $\mathrm{x}$-ray coherence length. It is convenient to use the uncorrected peak intensity $\left(I_{n c}\right)$ before the edge at an energy $E_{0}$ as a reference where we note $\mu_{0}=\bar{\mu}\left(E_{0}\right)$. The final expression for the isotropic case is

$$
I=e^{a\left(\mu_{0}-\bar{\mu}\right) \delta}\left(\frac{\mu_{0}}{\bar{\mu}}\right)^{n} I_{n c},
$$

where $n=1$ or 2 for peak or integrated intensity measurements, respectively.

We now consider in the model the effect of including birefringence from the anisotropic crystal, which necessitates a detailed description of the photon polarization. Here, we adopt standard results ${ }^{3,14,16}$ whereby the polarization state is expressed in terms of a linear combination of basis states corresponding to the polarization parallel $(\pi)$ and perpendicular $(\sigma)$ to the scattering plane. The unit-cell scattering amplitude for the four polarization permutations is given by the matrix

$$
F=\left(\begin{array}{ll}
F_{\sigma \sigma^{\prime}} & F_{\pi \sigma^{\prime}} \\
F_{\sigma \pi^{\prime}} & F_{\pi \pi^{\prime}}
\end{array}\right) .
$$

Just as the matrix $F$ operates on the incident beam to generate the scattered beam, we can write an expression for the action of the diffraction-based polarization analyzer. ${ }^{3}$ Because we assume a perfect analyzer, we use, as for the incoming polarization $\mathbf{P}$, a two-component vector representation, $\mathbf{A}^{+}=(1,0)$ or $\mathbf{A}^{+}=(0,1)$, to detect $\sigma^{\prime}$ and $\pi^{\prime}$ components, respectively.

In the kinematic approximation, diffracted intensity is then given by

$$
I \propto\left|\mathbf{A}^{+} F P\right|^{2},
$$

where the + symbolizes the conjugate transpose.
We have so far accounted for the polarization dependence of scattering from a thin layer within the sample, followed by a polarization analysis. To include absorption, dichroism, and birefringence from the the crystal before and after the scattering layer, we define the transmittance matrix

$$
T=e^{-\frac{1}{4}\left(\mu_{\sigma \sigma}+\mu_{\pi \pi}\right) l}\left(\begin{array}{ll}
T_{\sigma \sigma} & T_{\pi \sigma} \\
T_{\sigma \pi} & T_{\pi \pi}
\end{array}\right),
$$

with

$$
\begin{aligned}
& T_{\sigma \sigma}=\cosh (\tau l)+\frac{\mu_{\pi \pi}-\mu_{\sigma \sigma}}{4 \tau} \sinh (\tau l), \\
& T_{\sigma \pi}=-\frac{\mu_{\sigma \pi}}{2 \tau} \sinh (\tau l),
\end{aligned}
$$

where $\tau=\frac{1}{4} \sqrt{\left(\mu_{\sigma \sigma}-\mu_{\pi \pi}\right)^{2}+4 \mu_{\sigma \pi} \mu_{\pi \sigma}} \cdot \mu=\mu^{\prime}+i \mu^{\prime \prime}$ is the complex linear absorption coefficient. Its real part gives the absorption and dichroism (we have already noted that $\bar{\mu}$ is its spherical average) and the imaginary part, given by the corresponding Kramers-Kronig transform of the real spectrum, is responsible for birefringence. The indices symbolize the polarization dependence. $T_{\pi \sigma}$ and $T_{\pi \pi}$ are obtained by a simple permutation of the indexes from Eq. (5). Off-diagonal terms, which drive the polarization rotation, are always present for noncubic materials unless the propagation direction coincides with a principal axis, i.e., the wave is an eigenstate of the transmittance matrix. (Note also that for a magnetic material one has $\mu_{\sigma \pi} \neq \mu_{\pi \sigma}$.)

Ultimately, the observed intensity is obtained by multiplying the four matrices that modify the x-ray wave, leading to a simple expression for an arbitrary incident polarization condition:

$$
I=\frac{C \lambda^{3}}{v^{2} \sin 2 \theta_{B}} \mu_{0} \int_{0}^{\infty} d z\left|\mathbf{A}^{+} T_{s} F T_{i} \mathbf{P}\right|^{2},
$$

where $C, \lambda$, and $v$ are, respectively, a constant, the x-ray wavelength, and the unit-cell volume. ${ }^{17}$ From this formula, one finds that even with a forbidden $F_{\sigma \sigma^{\prime}}=0$, one can get a $\sigma \sigma^{\prime}$ intensity component because, at the depth $z$, the incoming $\sigma$ polarized wave is rotated and it acquires a $\pi$ component. For an isotropic material, the integral on the right gives, for the $\sigma \sigma^{\prime}$ channel, $\left|F_{\sigma \sigma^{\prime}}\right|^{2} / \bar{\mu}$. As discussed previously, we also eventually multiply by the prefactor $\mu_{0} / \bar{\mu}$ for measurement of the peak intensity and by $e^{a\left(\mu_{0}-\bar{\mu}\right) \delta}$ to take into account a disordered layer.

Calculations of the various spectra and azimuthal scans (rotations about the scattering vector) are fully relativistic and thus include the spin orbit in both the core and valence states. In the antiferromagnetic supercell, there are two nonequivalent $\mathrm{Cu}$ sites of multiplicity 8 (without magnetism all the $\mathrm{Cu}$ atoms are equivalent). The calculation of the unit-cell structure factor of the $(1 / 2,0,-1 / 2)$ reflection is done using the space and magnetic symmetry:

$$
F=2\left(1+m_{b}\right)(1-T)\left(f_{\mathrm{Cu} 1}+i f_{\mathrm{Cu} 2}\right),
$$

where $f_{\mathrm{Cu} 1}$ and $f_{\mathrm{Cu} 2}$ are the atomic scattering amplitudes, $m_{b}$ is the mirror symmetry operation perpendicular to $b$, and $T$ is the time reversal operator. The $1-T$ factor makes that the reflection is magnetic. The atomic scattering amplitudes are 
given by

$$
f_{\mathrm{Cu}}^{*}=\frac{m_{e}}{\hbar^{2}} \sum_{f, g} \frac{\left(E_{f}-E_{g}\right)^{2}\left\langle g\left|o_{s}^{*}\right| f\right\rangle\left\langle f\left|o_{i}\right| g\right\rangle}{\hbar \omega-\left(E_{f}-E_{g}\right)+i \Gamma / 2},
$$

where $m_{e}, f$, and $g$ are the electron mass and the unoccupied and core states. $E_{f}, E_{g}, \hbar \omega$, and $\Gamma$ are the $f$ and $g$ state energies, the photon energy, and the broadening. $o_{i}$ and $o_{s}$ are the transition operators corresponding to the incoming and scattered waves given by

$$
o=\boldsymbol{\epsilon} \cdot \mathbf{r}\left(1+\frac{i}{2} \mathbf{k} \cdot \mathbf{r}\right)+\beta(\mathbf{k} \times \boldsymbol{\epsilon}) \cdot(\mathbf{L}+2 \mathbf{S})+\cdots,
$$

where $\boldsymbol{\epsilon}$ and $\mathbf{k}$ are the photon polarization and wave vector, $\beta=-\hbar /\left[2 m_{e}\left(E_{f}-E_{g}\right)\right]$, and $\mathbf{L}$ and $\mathbf{S}$ are the angular momentum and spin operators. The first and second terms give the so-called electric $E 1$ and $E 2$ transitions; the third one gives the magnetic $M 1$ transition. $E 1, E 2$, and $M 1$ are governed by different selection rules on the probed valence states, making resonant $\mathrm{x}$-ray diffraction a unique probe of the electromagnetic properties of crystals. Equation (8) produces a series of pure and mixed multipole transitions. The atomic scattering amplitudes are calculated within a tensorial framework considering successively all the transition channels $E 1 E 1, E 1 E 2, E 2 E 2, M 1 M 1$, and $E 1 M 1$. After some algebra, Eq. (8) for the $E 1 E 1$ channel shows that the signal is proportional to the component of the atomic magnetic moment along the $b$ crystal axis. In the same way $E 1 E 2$ and $E 1 M 1$ depend on the toroidal moment around the same atoms. The purpose here is also to quantify all these terms.

As shown in Fig. 2, we find an excellent agreement for both the x-ray absorption near-edge structure (XANES)

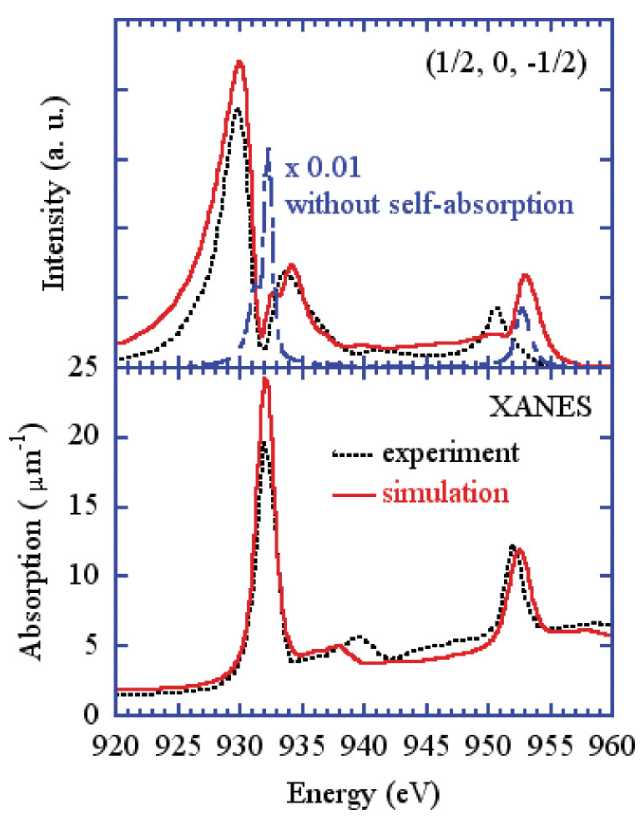

FIG. 2. (Color online) Top: Spectra of the $(1 / 2,0,-1 / 2)$ magnetic reflection in $\mathrm{CuO}$. The self-absorption is responsible for the vanishing intensity at the energy of the maximum of absorption. The reflection without self-absorption correction is also shown, divided by 100 . Data taken from Ref. 11, but the RXD spectra are provided by the same authors from an experiment done after publication.

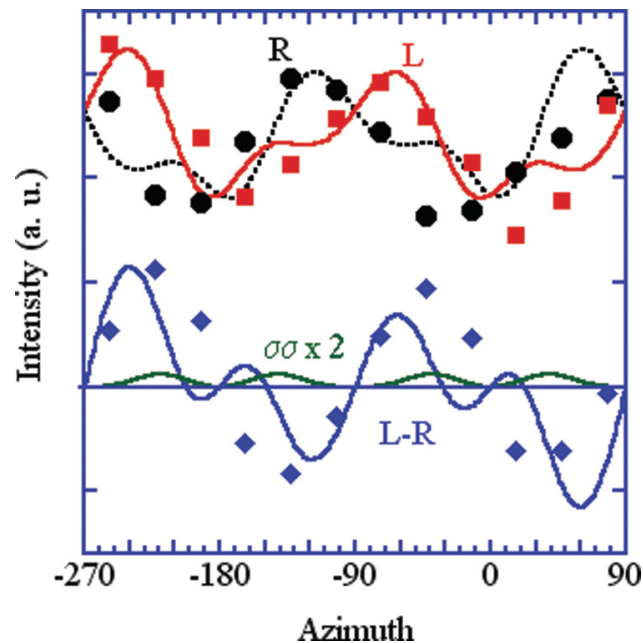

FIG. 3. (Color online) Azimuthal scan of the $(1 / 2,0,-1 / 2)$ magnetic reflection in $\mathrm{CuO}$ at $930 \mathrm{eV}$. The points are the experiment and the lines the simulation. Left and right polarizations are shown with their difference at the bottom. Note the good agreement in amplitude. The nonzero $\sigma \sigma^{\prime}$ signal is also shown multiplied by 2 . Data from Ref. 11.

and the diffraction peak spectrum shapes using only the $E 1 E 1$ channel, including birefringence. Since the spectra were obtained by measuring the peak intensity rather than area, the double correction is necessary to reproduce the experiment. We also add a $20 \mathrm{~nm}$ thick disordered layer on top of the surface which is parallel to the diffraction plane $(\alpha=0)$. The spectra appear qualitatively different after absorption correction, which produces a double peak shape with a maximum at $930 \mathrm{eV}$ that is not related to any feature of the resonant scattering amplitude.

In Fig. 3, we reproduce the azimuthal scan recorded at $930 \mathrm{eV}$ also only with the $E 1 E 1$ channel and the birefringence effect. Here the experimental points were recorded by integration so we apply only the single correction, i.e., without multiplying the intensity by $\mu_{0} / \bar{\mu}$. Without birefringence, incident beams with left and right circular polarization would produce identical spectra. We observe a strong difference, and reproduce not only the general shape but also the magnitude of the phenomenon. The difference between left and right circular polarization is $30 \%$ of the total intensity-exactly what we obtain from the simulation. This provides, perhaps, the most compelling evidence that the explanation in terms of birefringence is valid. In the figure we also show the calculated $\sigma \sigma^{\prime}$ intensity, which is clearly not zero. At $\psi=-43^{\circ}$, it is $5 \%$ of the intensity observed with $\pi \sigma^{\prime}$ polarization, and what remains anyway is four times smaller than in the experiment.

In Fig. 4, we show the change in amplitude with the azimuth around the $L_{3}$ edge. We observe the same effect in the experiment ${ }^{11}$ in spite of the fact that at $17^{\circ}$ the change in the relative intensity of the features is not complete, requiring a larger angle of $60^{\circ}$. This is consistent with the results from the azimuthal dependence, where we observe a poorest agreement at around $20^{\circ}$. Notwithstanding this discrepancy, we obtain very good agreement in both shape and magnitude. 


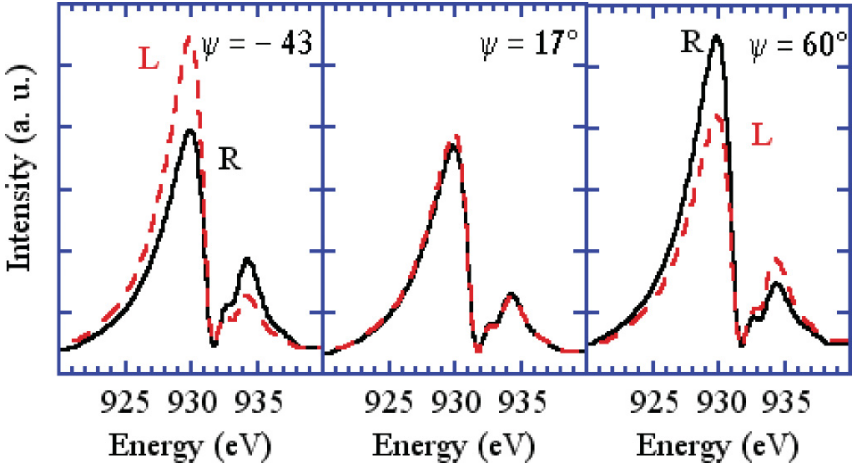

FIG. 4. (Color online) Spectra of the $(1 / 2,0,-1 / 2)$ magnetic reflection in $\mathrm{CuO}$ for incoming right $(\mathrm{R})$ and left $(\mathrm{L})$ polarizations and at different azimuths. We observe the same change as in the experiment of the relative amplitudes of the two main features vs the angle. Top: Data from Ref. 11.

To complete our analysis, we have performed simulations including the $E 1 M 1$ and the other contributions. These were found to play a very minor role compared to birefringence. We found the $E 1 M 1$ contribution to be $10^{-4}$ smaller in amplitude than the $E 1 E 1$ component. $E 1 E 2$ and $E 2 E 2$ are, respectively,
$3 \times 10^{-3}$ and $2 \times 10^{-4}$ smaller than $E 1 E 1$. Moreover, these amplitudes must be squared to obtain intensities. With the simulations being monoelectronic, it could be that the calculation of the toroidal electronic current corresponding to the so-called anapole is underestimated. Nevertheless its contribution is so small and the birefringence effect so large that there can be little doubt that it is the dominant physics in the reported measurements.

We have demonstrated that birefringence can play a major role in resonant diffraction experiments. We have proven that, even in the $\mathrm{x}$-ray energy range, this effect cannot always be neglected and should be modeled precisely. In the case of $\mathrm{CuO}$ we find no evidence for the existence of orbital currents. $A b$ initio simulations are absolutely necessary to interpret both the energy spectra, and azimuthal and polarization dependences. Resonant x-ray diffraction is a powerful tool, sensitive to very weak scattering phenomena and thus provides a way to measure them. Identification of $E 1 M 1$ contributions with confidence is likely to require experiments where competing effects, such as birefringence, can be ruled out.

We thank Stephen Lovesey, Valerio Scagnoli, and Urs Staub for helpful discussions and for providing the data. *yves.joly@grenoble.cnrs.fr

${ }^{1}$ D. H. Templeton and L. K. Templeton, Phys. Rev. B 40, 6505 (1989).

${ }^{2}$ M. Elzo et al., J. Magn. Magn. Mater. 324, 105 (2012).

${ }^{3}$ B. A. Palmer et al., J. Phys. Chem. Lett. 2, 2346 (2011).

${ }^{4}$ M. Sauvage, C. Malgrange, and J.-F. Petroff, J. Appl. Crystallogr. 16, 14 (1983).

${ }^{5}$ K. Okitsu et al., Proc. Soc. Photo-Opt. Instrum. Eng. 2873, 100 (1996).

${ }^{6}$ H.-C. Mertins, P. M. Oppeneer, S. Valencia, W. Gudat, F. Senf, and P. R. Bressler, Phys. Rev. B 70, 235106 (2004).

${ }^{7}$ S. Åsbrink and A. Waskowska, J. Phys.: Condens. Matter 3, 8175 (1991).

${ }^{8}$ T. Kimura et al., Nat. Mater. 7, 291 (2008).
${ }^{9}$ J. B. Forsyth et al., J. Phys. C 21, 2917 (1988).

${ }^{10}$ B. X. Yang, T. R. Thurston, J. M. Tranquada, and G. Shirane, Phys. Rev. B 39, 4343 (1989).

${ }^{11}$ V. Scagnoli et al., Science 332, 696 (2011).

${ }^{12}$ M. E. Simon and C. M. Varma, Phys. Rev. Lett. 89, 247003 (2002).

${ }^{13}$ W. B. Wu, D. J. Huang, J. Okamoto, S. W. Huang, Y. Sekio, T. Kimura, and C. T. Chen, Phys. Rev. B 81, 172409 (2010).

${ }^{14}$ S. P. Collins and S. W. Lovesey, J. Synchrotron Radiat. 8, 1065 (2001).

${ }^{15}$ O. Bunau and Y. Joly, J. Phys.: Condens. Matter 21, 345501 (2009); http://www.neel.cnrs.fr/fdmnes.

${ }^{16}$ S. W. Lovesey, Rep. Prog. Phys. 56, 257 (1993).

${ }^{17}$ B. E. Warren, X-ray Diffraction (Dover, New York, 1992). 